\title{
NATURAL HISTORY NOTE \\ Confirmada en Zamora (España) una colonia de apareamiento de nóctulo mediano, Nyctalus noctula (Schreber, 1774) en el límite más occidental de su distribución
}

\author{
Manuel Fabio Flechoso del Cueto ${ }^{1 *}$, Antonio San Martín González ${ }^{1}$, Pablo Santos Redín ${ }^{1}$, Gonzalo \\ Alarcos Izquierdo², Ricardo Jara Acevedo 3
}

\author{
${ }^{1}$ Servicio Territorial de Medio Ambiente \\ de Zamora. Junta de Castilla y León. C/ \\ Leopoldo Alas Clarín 4, 49071 Zamora, \\ Spain \\ ${ }^{2}$ C/ Castañal 10, 49321 Robleda- \\ Cervantes. Zamora, Spain \\ ${ }^{3}$ Centro de Investigación del Cáncer. \\ Avda. Universidad de Coimbra s/n. \\ 37007, Salamanca, Spain \\ *Corresponding author: \\ fabioflechoso@hotmail.com \\ DOI: https://doi.org/10.14709/ \\ BarbJ.13.1.2020.04 \\ Palabras clave: Catálogo Español de \\ Especies Amenazadas, colonia de \\ apareamiento, conservación, Natura \\ 2000, península Ibérica. \\ Keywords: Catálogo Español de \\ Especies Amenazadas, mating colony, \\ conservation, Natura 2000, Iberian \\ peninsula. \\ received: October, 23rd 2019 \\ accepted: March, 23rd 2020
}

\begin{abstract}
RESUMEN
El nóctulo mediano (Nyctalus noctula) es un murciélago forestal migrador, clasificado como "preocupación menor" por la IUCN, y como "vulnerable" en el Catálogo Español de Especies Amenazadas debido a su escasez regional. En la península Ibérica únicamente se tiene constancia de seis agrupaciones de nóctulos medianos, en Castilla y León, Navarra, Zaragoza, Lleida y Madrid. Entre los veranos de 2018 y 2019 se identificaron al menos 18 ejemplares y un mínimo de diez refugios utilizados por esta especie en una alameda del término municipal de Almaraz de Duero (Zamora, España) ubicada a orillas del río Duero. Los refugios se localizaron mediante la detección de llamadas sociales emitidas por los machos, todos ellos en nidos abandonados de pícidos (Picidae) y construidos sobre álamos blancos (Populus $a l b a)$. La altura de los nidos ocupados varió entre los 8 y los 14 metros. Además, en septiembre de 2019 se capturó un macho, confirmando las observaciones acústicas. El tamaño de los grupos que utilizaban los refugios varió entre individuos solitarios y un máximo de 17 ejemplares observados en septiembre de 2019. Los datos obtenidos no permiten conocer la composición de los grupos, aunque las llamadas sociales son las típicas de cortejo, dato que confirmaría la existencia de al menos una colonia de apareamiento. La nueva localidad se encuentra a unos 300 kilómetros de las más cercanas conocidas (Almazán y Aranjuez), y amplía hacia el oeste el área de distribución de las agrupaciones de nóctulos medianos tanto en la península Ibérica como a nivel europeo y mundial. La alameda estudiada se encuentra protegida al estar incluida en el espacio Natura 2000, ZEC Cañones del Duero (ES4190102) y ZEPA Cañones del Duero (ES0000206), considerándose un bosque muy relevante para la especie, cuya conservación es prioritaria.
\end{abstract}


distribution area of common noctule clusters on the Iberian Peninsula, Europe and worldwide. The poplar forest studied is protected by being included in the Natura 2000 area, ZEC Cañones del Duero (ES4190102) and ZEPA Cañones del Duero (ES0000206), being considered a very important forest for the species, whose conservation is a priority.

El nóctulo mediano (Nyctalus noctula) es un quiróptero forestal migrador, de tamaño grande comparado con otras especies europeas (Strelkov 2000, Dietz et al. 2009). Según la IUCN está catalogado como preocupación menor (LC) (Csorba \& Hutson 2016), aunque debido a su escasez en España se incluye como especie vulnerable $(\mathrm{Vu})$ en el Catálogo Español de Especies Amenazadas (Real Decreto 139/2011 de 4 de febrero). Su distribución a nivel mundial comprende desde la península Ibérica hasta Asia ocupando prácticamente toda Europa, y desde los países mediterráneos hasta el sur de Escandinavia (Dietz et al. 2009, Csorba \& Hutson 2016). Pese a su amplia distribución, sus citas en la península son muy escasas y apenas se han realizado observaciones de colonias sedentarias, hasta el punto de que en la península Ibérica solo se conocen seis pequeñas agrupaciones de esta especie, distribuidas por el centro y norte (Fig. 1): Aranjuez en Madrid (Ruedi et al. 1998), Pamplona y Sangüesa en Navarra (Alcalde 1999), Lleida (Camprodon \& Guixé 2013), Nuévalos en Zaragoza (Lorente et al. 2014) y Almazán en Soria (Flechoso et al. 2018). Únicamente la primera es reproductora, mientras que las demás están formadas exclusivamente por machos sedentarios en verano, que se ven acompañados de hembras desde finales de agosto hasta mediados de otoño constituyendo colonias de apareamiento. Durante el periodo de apareamiento los machos en celo son territoriales y emiten potentes vocalizaciones desde sus refugios, lo que facilita su localización (Kronwitter 1988, Alcalde 2008). El origen de las hembras que aparecen en estas agrupaciones de apareamiento a lo largo de otoño y pasan el invierno en la península es desconocido (Alcalde 2008), aunque es probable que procedan de colonias reproductoras asentadas en el continente europeo, como se ha comprobado con hembras de nóctulo pequeño, Nyctalus leisleri (Ohlendorf et al. 2000, Wohlgemuth et al. 2004, Alcalde et al. 2013b) y con N. lasiopterus (Guixé \& Camprodon 2018). En septiembre del año 2013 se grabaron algunas llamadas sociales en el valle del río Sabor al norte de Portugal, aunque su presencia como agrupación sedentaria o de apareamiento no ha podido ser confirmada mediante la captura de ningún ejemplar (Barros et al. 2014).

En esta nota presentamos la cita de una nueva colonia de apareamiento de nóctulo mediano, Nyctalus noctula (Schreber, 1774) hallada en una alameda junto al río Duero y localizada en el límite más occidental de su distribución.

La alameda objeto de estudio se encuentra en el margen derecho del río Duero, aguas abajo de la ciudad de Zamora de la que dista unos $10 \mathrm{~km}$ en línea recta, ocupando una superficie aproximada de 23,6 ha. Se trata de una alameda natural y madura que forma parte del bosque de ribera, con pies de diferentes edades y estrato arbustivo desarrollado. Debido a la competencia por la luz solar los árboles presentan alturas superiores a ocho metros en la mayoría de los casos pudiendo alcanzar hasta los 20 metros de altura. Se encuentra localizada en el término municipal de Almaraz de Duero, dentro del espacio Natura 2000 ZEC Cañones del Duero (ES4190102) y ZEPA Cañones del Duero (ES0000206), a unos $610 \mathrm{msnm}$ y tiene una longitud de unos $3.300 \mathrm{~m}$ lineales (Fig. 1 y 2). Las especies arbóreas más abundantes son álamos (Populus alba) y chopos negros (Populus nigra), aunque también hay sauces (Salix $s p$.) y diversas especies arbustivas. Los árboles de mayor porte contienen gran abundancia de nidos de pícidos (Fam. Picidae).

Durante los periodos julio-septiembre de 2018 y junioseptiembre de 2019 identificamos refugios de murciélagos mediante transectos realizados al atardecer, gracias a la escucha de llamadas sociales producidas por nóctulos (Alcalde 2008, Flechoso et al. 2018). También realizamos grabaciones de ultrasonidos de animales en vuelo en tiempo expandido utilizando un micrófono de ultrasonidos Pettersson M 500-384 conectado al teléfono móvil, que posteriormente analizamos mediante el programa Bat Explorer (Elekon, Luzern, Switzerland). Observamos ejemplares en vuelo que por dimensiones, tipo de vuelo y silueta podrían corresponder con las dos especies de tamaño grande del género Nyctalus ( $N$. lasiopterus y $N$. noctula). Al anochecer utilizamos diferentes métodos de captura para intentar capturar algunos ejemplares: redes, trampas de arpa y un dispositivo fabricado con una trampa tipo Sherman de aluminio para micromamíferos (233 x 90 x

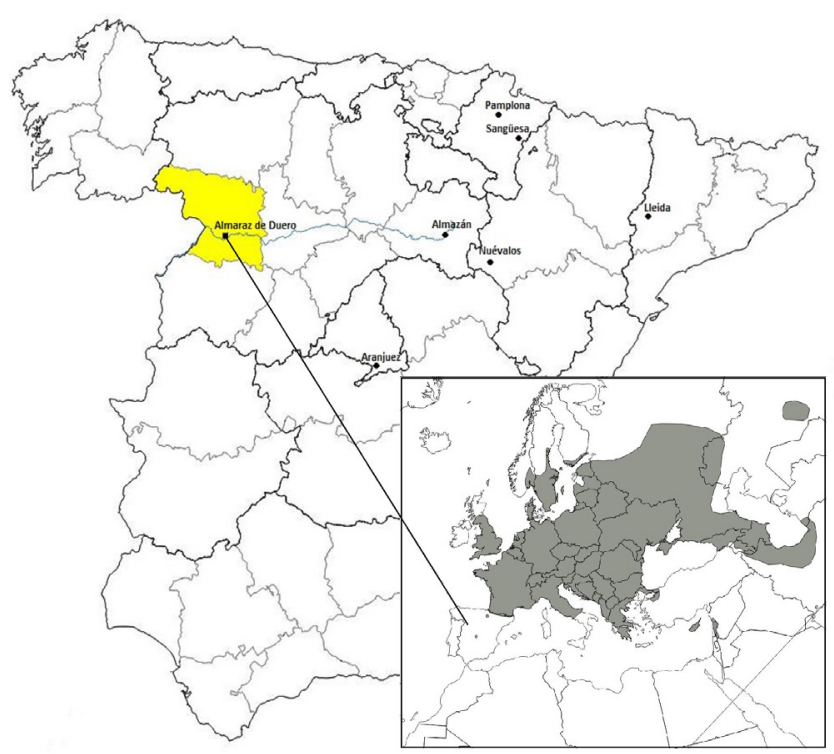

Fig. 1 - Rango de distribución de N. noctula en Europa (sombreado) según UICN (Csorba \& Hutson 2016) y localización de la colonia de Almaraz de Duero, provincia de Zamora (en amarillo), junto al Río Duero (en azul) y localización de las demás agrupaciones conocidas en España. Mapa modificado a partir de la base cartográfica disponible en http://centrodedescargas.cnig.es y https://www. iucnredlist.org/species. 
$75 \mathrm{~mm}$ ) acoplada a una pértiga de cuatro metros mediante cinta adhesiva (Fig. 3). Todas las trampas fueron colocadas a la salida de los refugios, en ocasiones con la ayuda de una escalera de 5 metros de longitud.

En julio de 2018 detectamos llamadas sociales típicas de $N$. noctula en un nido abandonado de pícido, situado en un álamo a unos 10 metros de altura. Adicionalmente realizamos grabaciones de ultrasonidos emitidas por los ejemplares en vuelo en el exterior de los refugios, y tras su análisis y siguiendo el método desarrollado por Barataud (2012), comprobamos que efectivamente correspondían a $N$. noctula (frecuencia de máxima intensidad alrededor de los 19 kHz y duración de unos 16 ms). Además, observamos dos agujeros de pícidos con el mismo patrón de desgaste y coloración descrito en otras ocasiones, aparentemente ocupados por esta especie (Flechoso et al. 2018). Mediante observación directa comprobamos que de uno de ellos salió un murciélago de tamaño relativamente grande que por su silueta y forma de volar podría corresponder con la especie citada.

En septiembre del mismo año detectamos un nuevo ejemplar emitiendo llamadas sociales dentro de un refugio en otra parte de la zona de estudio. Posteriormente intentamos capturar un ejemplar en uno de los refugios descritos mediante una trampa de arpa sin resultados positivos debido a la excesiva altura y dificultad para la colocación de la misma.

En junio de 2019 llevamos a cabo una nueva revisión de la alameda y localizamos tres nuevos nidos ocupados (según indicios externos) en los que oímos llamadas sociales en al menos uno de ellos. En julio visitamos otra parte de la zona de estudio sin detectar llamadas sociales en ningún punto aunque observamos varios individuos que por su silueta y forma de volar podrían corresponder con la especie citada.

El 30 de agosto intentamos capturar algún ejemplar colocando siete redes en diferentes agujeros de pícidos y observamos como de uno de ellos se escapó un ejemplar por un lateral del tronco. Además detectamos al menos cuatro nidos de pícidos con indicios de estar ocupados por nóctulos debido a las manchas existentes en el exterior pero que debido a la excesiva altura a la que se encontraban no pudieron ser trampeados.

El día 4 de septiembre visitamos de nuevo el mismo agujero donde se había escapado el ejemplar anterior. Este se encuentra a unos 8 metros de altura por lo que para acceder a él utilizamos una escalera y un dispositivo formado por una trampa tipo Sherman unida a una pértiga. A la entrada de la trampa se fijó una tira de espuma para sellar y ajustar mejor el dispositivo al agujero y la pértiga se ajustó al tronco del árbol mediante bridas de plástico. Con este método conseguimos capturar un individuo del cual se tomaron medidas biométricas de su antebrazo con el objetivo de corroborar los datos de las grabaciones de ultrasonidos obtenidas, confirmándose la especie como N. noctula (Fig. 4). El animal capturado, macho, con un antebrazo de $52,8 \mathrm{~mm}$, emitió potentes sonidos sociales durante su examen, presentó glándulas bucales desarrolladas y testículos escrotales. Además, observamos

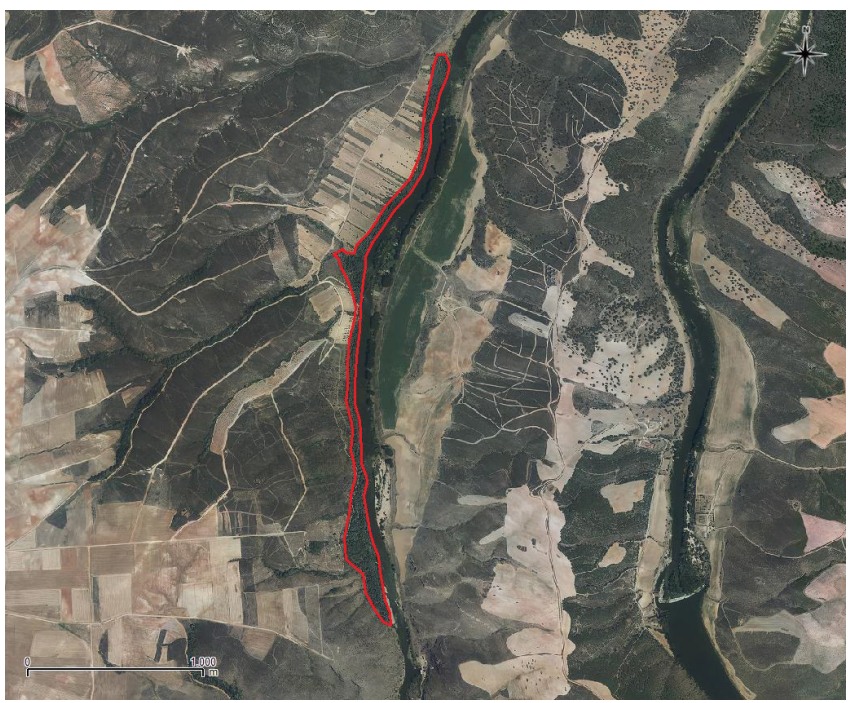

Fig. 2 - Plano de la zona de estudio en Almaraz de Duero donde se han encontrado los refugios de $N$. noctula. Ortofotografía PNOA 2017 modificada a partir de la base cartográfica disponible en http://centrodedescargas.cnig.es/.

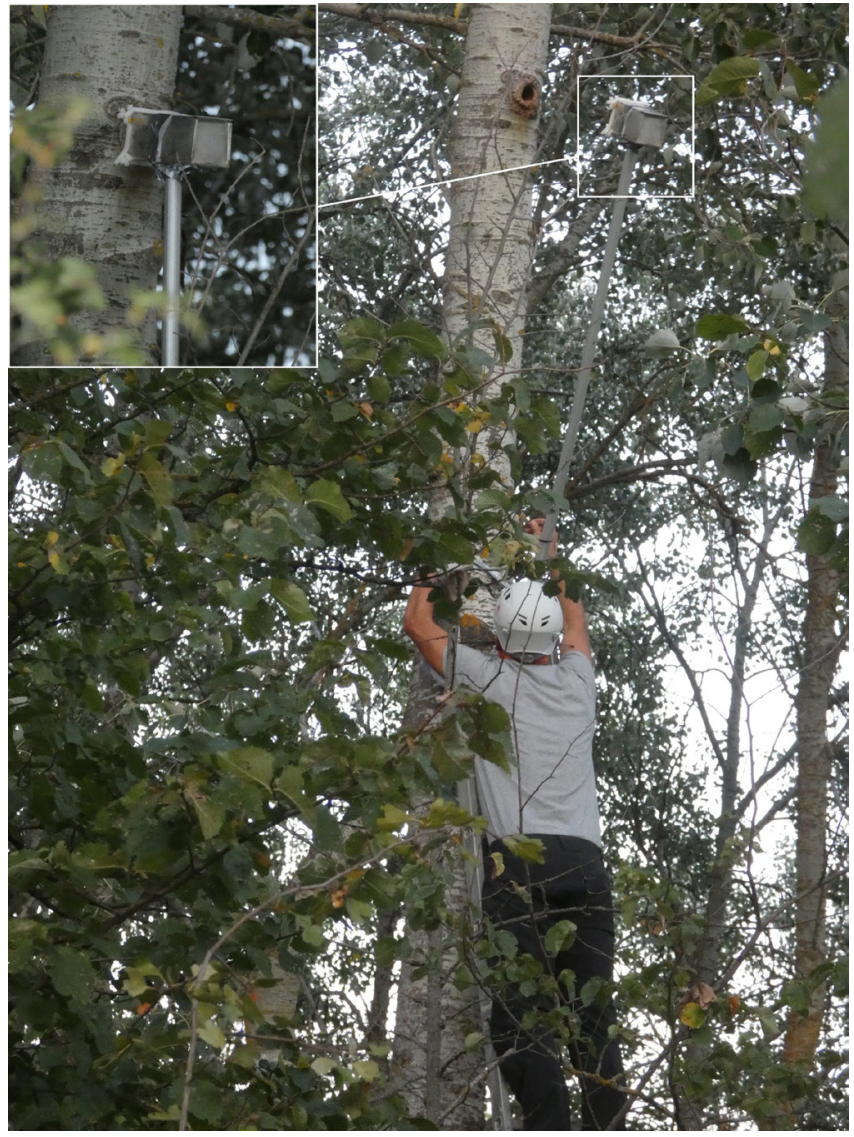

Fig. 3 - Trampa empleada para la captura del ejemplar de nóctulo mediano (N. noctula) (Almaraz de Duero). Autor Ricardo Jara.

como de un agujero situado a unos tres metros de altura por encima del mismo árbol, salieron en un intervalo de unos 10 minutos (entre las 21:00 y 21:10 horas) 17 ejemplares que por tamaño y tipo de reclamo podrían ser atribuibles a la misma especie. Se desconoce si se trata de un agujero doble o son dos independientes, no existiendo más agujeros en ese árbol. Adicionalmente se escucharon Ilamadas sociales en ambos refugios antes de la salida de los murciélagos. Los nóctulos del refugio superior realizaron diversas pasadas 
cercanas al refugio del macho emitiendo más llamadas sociales durante unos minutos antes de abandonar el lugar con un comportamiento similar al descrito por Flechoso et al. (2018). El ejemplar capturado, después de ser medido, se liberó inmediatamente en el mismo lugar para causarle las menores molestias posibles.

Por último, el día 12 de septiembre visitamos de nuevo la zona a la misma hora y localizamos tres nuevos refugios ocupados, de los que observamos salir al menos tres ejemplares diferentes (uno de cada refugio). Posteriormente a la salida de estos observamos en vuelo por lo menos siete ejemplares diferentes. Los datos más importantes se pueden apreciar a modo de resumen en la Tabla 1.

Esta nueva localidad se encuentra a unos 300 kilómetros de las más cercanas conocidas con poblaciones estables (Almazán y Aranjuez), y amplía hacia el oeste el área de distribución de las agrupaciones de nóctulos medianos tanto en la península Ibérica como a nivel mundial (Fig. 1) (Csorba \& Hutson 2016). La observación de ejemplares durante varios meses del año en dos años consecutivos, así como el aspecto de la boca de algunos refugios que se encuentran desgastados y con un tono pardo-amarillento, característico del paso de los murciélagos (Gebhard \& Bogdanowicz 2004), permite suponer que existe una colonia sedentaria que utiliza regularmente esta alameda y que por tanto su presencia no es ocasional. Además, estos nueve refugios se encuentran en árboles diferentes a excepción de uno de ellos que tiene dos refugios ocupados. La totalidad de los refugios ocupados hallados se encuentran entre los 8 y los 14 metros de altura, en el interior de nidos de pícidos y sobre $P$. alba.

Por los datos recogidos hasta la fecha, se desconoce si la colonia hallada muestra una fenología similar a las presentes en Navarra (Alcalde 2008), Aragón (Lorente et al. 2014) y Soria (Flechoso et al. 2018). La composición del grupo de 17 ejemplares saliendo de un mismo refugio contiguo al del macho capturado en septiembre de 2019 es una incógnita. Por una parte, en esta fecha es de esperar que haya comenzado el periodo de apareamiento y que los machos ya se encuentren dispersos por lo que es algo tarde para que sea una agrupación exclusiva de machos. Pero por otra parte el número de individuos del grupo es muy superior al de un harén normal (4-5 hembras) (Dietz et al. 2009). La población

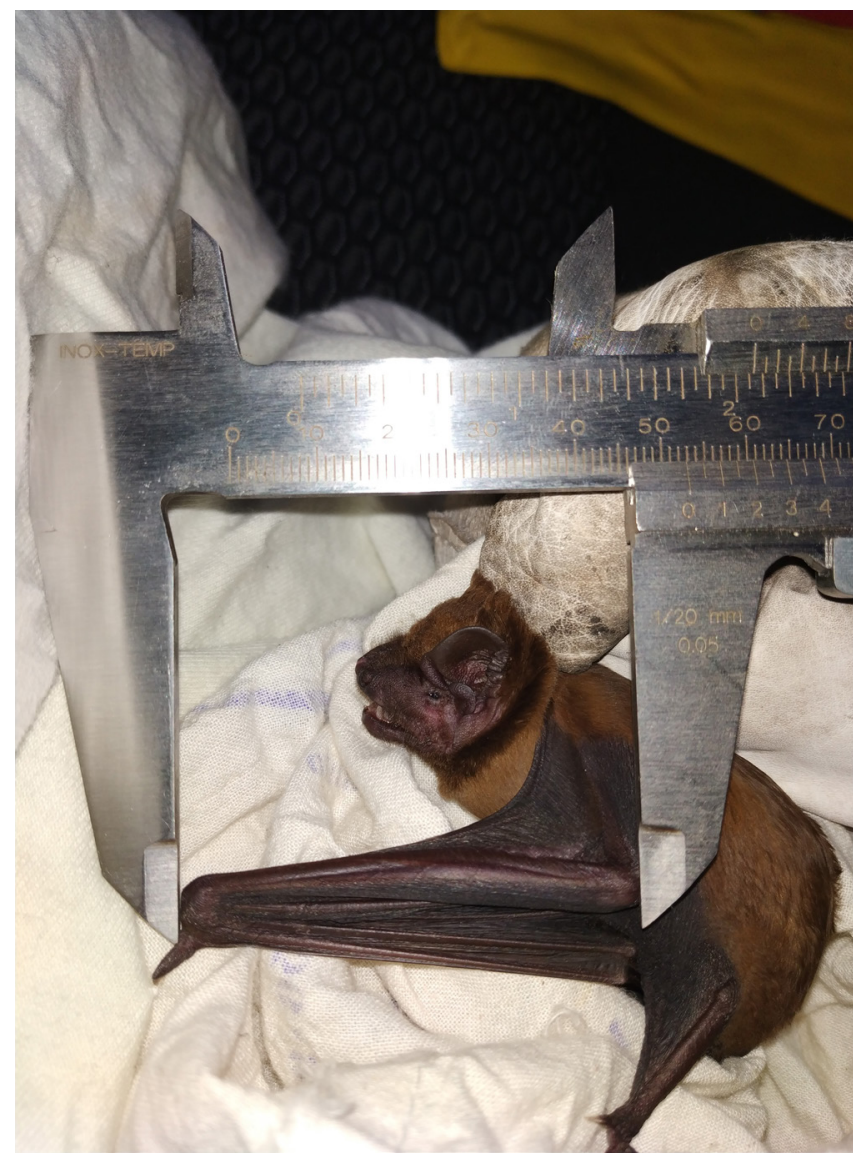

Fig. 4 - Nóctulo mediano ( $N$. noctula) fotografiado después de ser capturado mientras se tomaban medidas biométricas (Almaraz de Duero). Autor Antonio San Martín.

encontrada contiene un mínimo de 18 ejemplares en el mes de septiembre. No obstante, debido a la gran extensión de la alameda (Fig. 2), la gran abundancia de agujeros o nidos de pícidos y al haberse constatado la existencia de un mínimo de nueve refugios diferentes ocupados detectados durante todo el verano, esta población de nóctulo mediano debe contar con al menos varias decenas de individuos. La zona descrita plantea una elevada dificultad de muestreo ya que los nidos ocupados se encuentran a gran altura y los estratos inferiores a menudo tienen gran densidad de vegetación arbustiva.

Tabla 1 - Resumen de los datos obtenidos durante las diferentes visitas realizadas a la zona de estudio.

\begin{tabular}{|c|c|c|c|c|c|c|}
\hline Año & Mes & $\begin{array}{c}\text { Llamadas } \\
\text { sociales }\end{array}$ & $\begin{array}{l}\text { Grabaciones } \\
\text { ultrasonidos }\end{array}$ & $\begin{array}{l}\text { Refugios } \\
\text { ocupados }\end{array}$ & $\begin{array}{l}\text { Ejemplares } \\
\text { observados }\end{array}$ & $\begin{array}{l}\text { Individuos } \\
\text { capturados }\end{array}$ \\
\hline \multirow{2}{*}{2018} & Julio & $x$ & $x$ & 1 & & \\
\hline & Septiembre & $x$ & & & & \\
\hline \multirow{5}{*}{2019} & Junio & $x$ & $x$ & 1 & & \\
\hline & Julio & & & & varios & \\
\hline & Agosto & $x$ & $x$ & 4 & 1 & \\
\hline & Septiembre & $x$ & & & 18 & 1 \\
\hline & Septiembre & $x$ & & 3 & 7 & \\
\hline
\end{tabular}


A pesar de que las especies arbóreas utilizadas como refugios que han sido descritas y el hábitat en el que se encuentra la colonia presenta algunas similitudes, existen diferencias notables respecto a la mayor parte de las descritas para otras agrupaciones de esta especie en España, como las de Aranjuez (Ruedi et al. 1998), Pamplona y Sangüesa (Alcalde 1999) o Almazán (Flechoso et al. 2018). En este caso no se trata de un parque urbano con árboles frondosos de gran tamaño y con abundantes oquedades, cercanos a un tramo lento de un río, sino de un bosque natural con mayor densidad de vegetación. Ello indica que a pesar de existir cierta preferencia de los nóctulos medianos por este tipo de hábitat (por el espacio amplio que necesitan para maniobrar) y debido a que cazan en espacios abiertos, no parece tan importante la inexistencia de un estrato arbustivo desarrollado o de una mayor densidad de pies de varias clases de edad. En cambio, parece clara la preferencia por tramos lentos de ríos de cierto caudal, siendo el álamo blanco uno de sus árboles preferidos donde se han reportado refugios más frecuentemente. No obstante, convendría localizar nuevas agrupaciones de este murciélago en zonas similares para seguir evaluando este aspecto.

En todo caso, la presencia de grupos estables residentes de nóctulos medianos exige una especial atención frente a claras o cortas de arbolado. Si algún árbol con huecos requiriese ser cortado por algún motivo de fuerza mayor, sería conveniente realizar revisiones previas para asegurarse de que no hay nóctulos en su interior. En todo caso sería imperativo adoptar medidas compensatorias a la eliminación de refugios naturales como la instalación de cajas-refugio como, por ejemplo, el modelo 2FN de Schwegler. Se ha comprobado positivamente la utilización de este modelo por esta especie en Pamplona (Alcalde et al. 2013a).

\section{AGRADECIMIENTOS}

Especialmente a Javier García-Oliva y a Juan Tomás Alcalde, por su asesoramiento durante los muestreos. Al Servicio Territorial de Medio Ambiente de Zamora por la obtención de la autorización y permiso de captura de los ejemplares. Por último, a nuestras familias por su comprensión al haber prescindido de nosotros durante las salidas nocturnas realizadas.

\section{REFERENCIAS}

ALCALDE, J. T. (1999). New ecological data on the noctule bat (Nyctalus noctula Schreber, 1774) (Chiroptera, Vespertilionidae) in two towns of Spain. Mammalia, 63(3): 273-280. https://doi.org/10.1515/mamm.1999.63.3.273

ALCALDE, J. T. (2008). El nóctulo mediano (Nyctalus noctula) en Pamplona. Biodiversidad Urbana de Pamplona. ed.: Ayuntamiento de Pamplona - Iruñeko Udala. Pamplona, España, 52 pp.

ALCALDE, J. T., CAMPION, D., FABO, J., MARÍN, F., ARTÁZCOZ, A., MARTínEZ, I. \& ANTÓN, I. (2013a). Ocupación de cajas-refugio por murciélagos en Navarra. Journal of Bat Research \& Conservation, 6(1): 35-45. https://doi. org/10.14709/BarbJ.6.1.2013.05
ALCALDE, J. T., IBAÑEZ, C., ANTÓN, I. \& NYSSEN, P. (2013b). First case of migration of a Leisler's bat (Nyctalus leisleri) between Spain and Belgium. Le Rhinolophe, 19: 87-88.

BARATAUD, M. (2012). Acoustic ecology of European bats: species identification, habitat studies and hunting behaviour. Collection Inventaires et biodiversité. ed.: Biotope, Mèze \& National Museum of Natural History. Paris, France, 352 pp.

BARROS, P., BRAZ, L., VALE-GONÇALVES, H. M. \& CABRAL, J. A. (2014). First records of Nyctalus noctula social calls in Portugal. Vespertilio, 17: 37-44.

CAMPRODON, J. \& GUIXÉ, D. (2013). Estado poblacional, selección de refugios y ecología espacial de las poblaciones de nóctulo grande (Nyctalus lasiopterus) y nóctulo mediano (Nyctalus noctula) en Cataluña. Journal of Bat Research \& Conservation, 6(1): 53-61. https://doi. org/10.14709/BarbJ.6.1.2013.07

CSORBA, G. \& HUTSON, A. M. (2016). Nyctalus noctula. The IUCN Red List of Threatened Species 2016: e.T14920A22015682. https://doi.org/10.2305/IUCN. UK.2016-2.RLTS.T14920A22015682.en

DIETZ, C., VON HELVERSEN, O. \& NILL, D. (2009). Bats of Britain, Europe and Northwest Africa. ed.: A\&C Black Publishers Ltd. London, United Kingdom, 400 pp.

FLECHOSO, M. F., FERNÁNDEZ, D. \& ALCALDE J. T. (2018). Primera colonia de nóctulos medianos (Nyctalus noctula Schreber, 1774) en Castilla y León (Norte de España). Journal of Bat Research \& Conservation, 11(1). https:// doi.org/10.14709/BarbJ.11.1.2018.02

GEBHARD, J. \& BOGDANOWICZ, W. (2004). Nyctalus noctula (Schreber, 1774) Großer Abendsegler. In: Handbuch der Säugetiere Europas. Fledertiere II. ed.: Niethammer, J. \& Krapp, F. Wiebelsheim, Germany, p.605-694.

GUIXÉ, D. \& CAMPRODON, J. (2018). Manual de conservación y seguimiento de los quirópteros forestales. ed.: Ministerio de Agricultura, Pesca y Alimentación; Ministerio para la Transición Ecológica. Madrid, España, 274 pp.

KRONWITTER, F. (1988). Population structure, habitat use and activity patterns of the noctule bat, Nyctalus noctula, revealed by radio-tracking. Myotis, 26: 23-85.

LORENTE, L., LLANA, C. \& SÁNCHEZ, J. M. (2014). Primera cita de nóctulo mediano (Nyctalus noctula) en Aragón (NE de España). Journal of Bat Research \& Conservation, 7(1): 19-21. https://doi.org/10.14709/BarbJ.7.1.2014.04

OHLENDORF, B., HECHT, B., STRASSBURG, D. \& AGIRREMENDI, P. (2000). Fernfund eines Kleinabendseglers (Nyctalus leisleri) in Spanien. Nyctalus, 7 (3): 239-242.

RUEDI, M., TUPINIER, Y. \& DE PAZ, O. (1998). First breeding record for the noctule bat (Nyctalus noctula) in the Iberian Peninsula. Mammalia, 62(2): 301-304. 
STRELKOV, P. (2000). Seasonal distribution of migratory bat species (Chiroptera, Vespertilionidae) in Eastern Europe and adjacent territories: nursing area. Myotis, 37: 7-25

WOHLGEMUTH, R., DEVRIENT, I., GARCÍA, A. \& HUTTERER, R. (2004). Long-distance flight of a lesser noctule (Nyctalus leisleri) after rehabilitation. Myotis, 41-42: 69-73. 